\section{PS5:104 LYMPHOPENIA IN SYSTEMIC LUPUS ERYTHEMATOSUS - A RETROSPECTIVE ANALYSIS FROM UNIDADE IMUNOLOGIA CLINICA (CENTRO HOSPITALAR E UNIVERSITÁRIO DO PORTO)}

T Fonseca, A Marinho, C Vasconcelos, F Farinha. Centro Hospitalar e Universitário do Porto - Unidade de Imunologia Clinica, Porto, Portugal

10.1136/lupus-2018-abstract.149

Systemic Lupus Erythematosus (SLE) has immune dysregulation, with lymphopenia being one of the most frequent clinical findings, namely the CD4 $\mathrm{T}$ cells. It has been associated not only to higher risk of infections, but also to disease activity and risk of flares.

The authors pretended to describe the prevalence of lymphopenia in SLE in a major centre of Immunology in Portugal. A retrospective analysis on the SLE patients was performed, with a longitudinal description of the subpopulations of lymphocytes, relating it with disease activity, organ involved, therapeutics and major infections.

The sample had 48 patients, mainly constituted by females $(85,4 \%)$, median age of 43 y. $62.5 \%$ had lymphopenia in the diagnosis. At the time of the most recent peripheral blood flow citometry $66,7 \%$ of the sample had lymphopenia, with different values of cytopenia according to lymphocytes subpopulations - T CD4: 77,4\%; T CD8: 75\%; B: 83,3\%; NK: 91,7\%. Severe T CD4 lymphopenia (below $200 \mathrm{uL}$ ) was present in $8,3 \%$ of the sample. $18,8 \%$ had severe flares (SLEDAI Index) and $90 \%$ of these had low T CD4 counts (below $700 \mathrm{uL}$ ). The majority of patients with T CD4 under $200 \mathrm{uL}$ were on severe flare. Higher frequency of corticosteroids and immunosuppressors, namely cyclophosphamide (12,5\%) was observed on the patients. 17 patients of the sample had sequential citometry analysis and a correlation between lymphopenia and activity has not observed. 17 cumulative infections were described, the majority $(70,6 \%)$ with lymphopenia. Although opportunistic infections (pulmonary aspergilosis, PML due to JC virus) were mainly seen on patients with $\mathrm{T}$ CD4 under $200 \mathrm{uL}$ it was not mandatory this condition on this sample - cryptococcal meningitis was described on a patient with $300 \mathrm{uL} \mathrm{T} \mathrm{CD4.}$

Lymphopenia was present in the majority of active lupus and T CD4 seems to correlate with severe flare. Lymphopenia seems to be a bystander on the evolution of the disease. Despite rare, unpredictable infections can appear on patients with T CD4 counts superior to $200 \mathrm{uL}$.

\section{PS5:105 HDL INHIBITS T CELL PROLIFERATION IS SLE}

${ }^{1} \mathrm{M}$ Fernandes Das Neves, ${ }^{1} \mathrm{C}$ Favas, ${ }^{2} \mathrm{~J}$ Batuca, ${ }^{3} \mathrm{EC}$ Jury, ${ }^{1,2}$ José Delgado Alves. ${ }^{1}$ Fernando Fonseca Hospital - Medicine 4 Department, Amadora, Portugal; ${ }^{2}$ NOVA Medical School CEDOC, Lisbon, Portugal; 'University College London - Centre for Rheumatology and Bloomsbury Rheumatology Unit, London, UK

\subsection{6/lupus-2018-abstract.150}

Purpose Systemic lupus erythematosus (SLE) is associated with dyslipidemia and increased cardiovascular risk. The SLE pattern is characterised by high plasma levels of low-density lipoproteins (LDL) and triglycerides and low levels of high-density lipoproteins (HDL). HDL is a complex plasma lipoprotein that is recognised for its protective role in atherosclerotic disease. It consists of an outer layer of lipids and apolipoproteins, with apolipoproteinA-1 (apoA-1) constituting $70 \%$ of the protein content, a triglyceride and cholesterol ester-rich core, and several enzymes. In addition to its antiatherogenic properties, mainly associated with reverse cholesterol transport from vessels, HDL has also anti-inflammatory properties that are not clearly understood. This work aims to show the effect of HDL on T lymphocyte proliferation.

Methods Peripheral blood mononuclear cells (PBMCs) were isolated from 7 SLE patients, with at least 4 SLICC/ACR classification criteria and normal serum lipid profiles, and 3 healthy donors. PBMCs were cultured with and without HDL (at the concentrations of 50,300 and $600 \mu \mathrm{g} / \mathrm{mL}$ ) before CD3 and CD28 stimulation. After 4 days in culture, T cell proliferation was measured by flow cytometry through Ki-67 staining. Regulator T cells (Tregs) phenotyping (CD4 +CD25+CD27FoxP3 +) was performed. The expression of the cholesterol transporter ABCA1 in $\mathrm{T}$ lymphocytes was also measured by flow cytometry.

Results HDL decreased T cell proliferation in a dose-dependent manner, with the biggest effect obtained with the physiologic concentration of $600 \mu \mathrm{g} / \mathrm{mL}$. The inhibition of $\mathrm{T}$ cell proliferation was more pronounced in SLE patients than in the healthy donors. SLE patients tend to have higher baseline $\mathrm{T}$ cell proliferation measured by ki-67 expression. There were no differences in the prevalence of Tregs among patients and healthy donors. The expression of ABCA1 on the surface of $\mathrm{T}$ lymphocytes was similar between groups.

Conclusions This study is the first demonstration of a regulatory effect of HDL on the adaptive immune system of SLE patients. Here we show that HDL can decrease T cell proliferation, which is not correlated with the expression of the ABCA1, the main cholesterol transporter to ApoA-1. We expect to further elucidate HDL effects on the immune system in future studies.

\section{PS5:106 LOW CIRCULATING BASOPHIL COUNTS IN BIOPSY- PROVEN ACTIVE LUPUS NEPHRITIS}

I Haddiya. Faculty of Medicine, University Mohamed First, Oujda, Morocco

10.1136/lupus-2018-abstract.151

The link between circulating basophil counts and renal pathology activity of lupus nephritis (LN) is not fully understood, although it's been observed that Basophils contribute to the immunopathogenesis of this disease.

Aim of the study To assess the relationship between low levels of circulating basophils counts and the activity of lupus nephritis.

Methods We performed a retrospective clinical study, 140 clinical and pathology samples from patients with biopsy-proven LN from were analysed. The renal activity and classification were evaluated according to renal pathology. SLE disease activity was scored using the SLE Disease Activity Index (SLEDAI).The correlations between circulating basophil counts and renal pathology activity index were assessed.

Results Mean age of our patients was 34.63 $\pm 12,7$ years old, $83 \%$ were females. Class III, IV and V lupus nephritis accounted for $21 \%, 58.7 \%$ and $11.2 \%$ respectively. Circulating basophil counts correlated with total systemic lupus erythematosus disease activity index (SLEDAI) score $(\mathrm{r}=-0.3)$, renal SLEDAI score $(\mathrm{r}=-0.32)$, activity index (AI) $\operatorname{score}(\mathrm{r}=-0.29)$, and renal histologic activity parameters $(\mathrm{p}<0.05$, respectively). 
Compared with systemic lupus erythematosus (SLE) non active LN patients, the active LN group showed lower basophil counts $(p=0.002)$. Moreover, Significant differences were found in circulating basophil counts among LN pathology classification groups $(p<0.05)$. Groups of classes III, IV were more likely to have lower circulating basophil counts when compared with the other classes $(p=0.047)$.

Conclusion According to these results circulating basophil counts might be an interesting marker for renal activity of LN.

\section{PS5:108 SEMAPHORIN3A: A BENEFICIAL THERAPY FOR LUPUS NEPHRITIS}

E Toubi, Z Vadasz. Bnai-Zion Medical Centre, The Technion Faculty of Medicine, Haifa, Israel

\subsection{6/lupus-2018-abstract.152}

Background The immune regulatory properties of semaphorin3A (sema3A) (both innate and adaptive) are well established in many in-vitro studies. When sema3A was incubated with active B cells from SLE patients, it could efficiently reduce the expression of TLR-9 in correlation with a significant reduction of relevant autoantibodies. The injection of sema3A to a mice model of rheumatoid arthritis was proven to be highly beneficial, both in attenuating clinical symptoms and in decreasing inflammatory mechanisms.

Objectives This study was designed in order to assess possible therapeutic benefits following the injection of sema3 A to NZB/W mice. Results The injection of sema3A to young mice (at week 12) before disease onset, delayed the appearance of proteinuria. Here, the median time to severe proteinuria was 110 days, 95\% CI: 88 to 131. However, in mice in which empty vector was injected, median time to severe proteinuria was 63 days, 95\% CI: 0 to 139). Sema3A treatment, reduced significantly renal damage, namely, it prevented the development of immune deposits in the glomeruli. When sema3A was injected at the onset of proteinuria, aiming to treat rather than to prevent disease in these mice, survival was significantly increased and the deterioration of proteinuria was significantly delayed. Conclusion Semaphorin3A is highly beneficial in reducing lupus nephritis in $\mathrm{NZB} / \mathrm{w}$ mice. It delays the appearance and deterioration of proteinuria, and increases survival rates in these mice. Further studies will establish the idea of applying sema3 A in the treatment of lupus nephritis.

\section{Poster session 6: Management and recommendations, diagnostic and classification criteria}

\section{PS6:109 ASSOCIATION OF MENTAL HEALTH RELATED QUALITY OF LIFE (HRQOL) AND SUBSEQUENT PHYSICAL FUNCTIONING IN SYSTEMIC LUPUS ERYTHEMATOSUS: ANALYSIS OF DATA FROM THE GERMAN LULA COHORT $2002-2013$}

${ }^{1} R$ Brinks, ${ }^{1} G$ Chehab, ${ }^{1} R$ Fischer-Betz, ${ }^{1}$ Jichter, ${ }^{1} R$ Willers, ${ }^{2} B$ Winkler-Rohlfing, ${ }^{1} \mathrm{M}$ Schneider. 'University Hospital Duesseldorf, Department and Hiller Research Unit for Rheumatology, Düsseldorf, Germany; ${ }^{2}$ Self help group for Lupus Erythematodes, Wuppertal, Germany

\subsection{6/lupus-2018-abstract.153}

Introduction Mental symptoms are a major problem in patients with systemic lupus erythematosus (SLE). From geriatric patients it is well-known that prevention of mental disorders leads to maintenance of physical functioning (PF).

Methods Data from the German Lupus-Langzeitstudie (LuLa) open cohort was used to explore the effect of self-reported mental HRQoL on PF. The ongoing LuLa study comprises patients from the German SLE self-help group. The current analysis uses data from 2002 to 2013. We looked for associations between the PF score (outcome) and the mental component summary (MCS) score (exposure) of the SF-12 questionnaire in one to four years preceding the outcome. Statistical analysis was accomplished by a linear mixed regression model (LMM) adjusting for the potential confounders age, sex, disease duration and disease activity as surveyed by the Systemic Lupus Activity Questionnaire score (SLAQ).

Results 1511 patients $(93.3 \%$ women) provided data with a total of 7841 person-years of observation. Median age at study entry was 46 (interquartile range: 37 to 57). Table 1 shows the regression coefficients of MCS (and 95\% confidence intervals, CI) estimated by the LMM without and with adjusting for age, sex, disease duration and SLAQ.

Conclusions We found evidence that self-reported mental HRQoL in patients with SLE is associated with PF in the subsequent years. The data indicate a 'window of opportunity' for treating mental symptoms of up to three years for maintenance of PF.

Abstract PS6:109 Table 1 Association between physical functioning (outcome) and mental health related quality of life (MCS) one to four years before the outcome

\begin{tabular}{|l|r|r|r|r|}
\hline \multirow{2}{*}{$\begin{array}{l}\text { Exposure: Mental component } \\
\text { summary score (MCS) }\end{array}$} & \multicolumn{2}{|c|}{ Crude estimates } & \multicolumn{2}{c|}{ Adjusted estimates } \\
\cline { 2 - 5 } & Estimate, 95\% CI & p-value & Estimate, 95\% CI & p-value \\
\hline 1 year ahead & $0.12,(0.07,0.17)$ & $<0.001$ & $0.09,(0.04,0.13)$ & $<0.001$ \\
2 years ahead & $0.12,(0.08,0.17)$ & $<0.001$ & $0.09,(0.04,0.13)$ & $<0.001$ \\
3 years ahead & $0.07,(0.02,0.12)$ & 0.009 & $0.06,(0.01,0.11)$ & 0.016 \\
4 years ahead & $0.02,(-0.04,0.07)$ & 0.564 & $0.02,(-0.03,0.08)$ & 0.395 \\
\hline
\end{tabular}

